\title{
Controversies
}

\section{Penicillamine Should Not Be Used as Initial Therapy in Wilson's Disease}

\author{
George J. Brewer, MD \\ University of Michigan Medical School, Ann Arbor, Michigan, U.S.A.
}

I feel honored to be asked by the Movement Disorders journal to contribute my thoughts to the controversy on how to treat patients with Wilson's disease, particularly during the initial period. I am doubly honored to cross swords on this topic with Professor John Walshe, who has contributed so much to the treatment of Wilson's disease. $^{1,2}$ As I have said in other settings, a whole generation of patients with Wilson's disease owe their lives to the pioneering work of Professor Walshe.

But then was then and now is now, and the two are not the same. Drugs that do wonderful things in one era, along with some damage, tend to be replaced by drugs in another era, drugs that still do wonderful things but with much less damage. That is exactly the situation we have at present with penicillamine. Its era is over. It is far too toxic to use when we have drugs that are just as effective and much safer.

One of the major problems with penicillamine occurs when it is used for the initial treatment of the patient presenting with neurologic disease. ${ }^{3}$ There is a major risk that the patient will be made permanently worse neurologically. Take the following case as an example of the disaster awaiting patient and physician when they use penicillamine in this situation. The patient was an attractive 27-year-old woman working on her master's degree. She developed depression, a mild dysarthria, and mild tremor, and was diagnosed as having Wilson's disease. She was started on $1.0 \mathrm{~g}$ penicillamine per day and over 4-8 weeks became much worse. Her dysarthria and tremor progressed, and she developed mild dystonia of

Received October 7, 1998. Accepted February 9, 1999.

Address correspondence and reprint requests to George J. Brewer, MD, University of Michigan Medical School, 1301 Catherine St., Ann Arbor, MI 48109-0618, U.S.A. the face, trunk, and limbs. Her dose of penicillamine was doubled. Over a 3-4-month period she worsened disastrously. Her dysarthria became severe, her posture changed because of the dystonia such that her back became continuously curved, and dystonia and tremor of her upper extremities worsened to the point where she was essentially unable to carry out even simple care tasks such as feeding herself. Her gait became distorted and uncomfortable, limiting her walking to very short stretches. Her face became grotesquely distorted with chronic drooling. Dysphagia developed and became severe enough to lead to frequent choking episodes, but the patient refused gastrostomy. This all happened 20 years ago, and despite many years of penicillamine therapy, bringing her copper status under good control, the patient has never recovered. She lives today crippled without hope of a meaningful social or working life. She has attempted suicide several times.

Was this patient's life destroyed by penicillamine? Some would argue that the real culprit was Wilson's disease and the toxicity of copper, not the drug designed to get rid of that copper. However, I can almost guarantee, as close as anyone can ever come to guaranteeing anything in medicine, that if I had that patient to treat now at the beginning of her presentation, she would have been able to subsequently live a healthy, essentially normal life. This tragedy was unavoidable then. It is inexcusable now.

Is penicillamine-induced worsening rare and this patient's course an isolated occurrence? Unfortunately not. Along with Carol Terry, then President of the Wilson's Disease Association, we did a retrospective survey of the Association members who had presented with neurologic symptoms and who had been treated with penicillamine. ${ }^{3}$ Of 27 respondents, 13 had shown initial deterioration, 
usually within 2-6 weeks of starting treatment, and six never recovered to their pre-penicillamine baseline. Most of this latter group were severely disabled, much like the patient we described here.

These data indicate that $50 \%$ of the time, patients of this type worsen on penicillamine, and $50 \%$ of that group, or almost $25 \%$ of the original group, are left with more serious disability than they started with. Retrospective surveys can be criticized, and I do not defend the numbers as being highly rigorous. However, the numbers clearly indicate that there is a high risk with penicillamine treatment to this type of patient, so much so that I often draw the analogy that the patient presenting neurologically has a ticking bomb within their head and penicillamine is just the drug to light the fuse. A roughly $25 \%$ probability of a disastrous result and ruined life is just too great a risk to take when today we have safer alternatives.

Some might argue that these deteriorating patients are simply going through the natural course of the disease, and that these worsenings were fated to occur because the damage had already set them in motion, no drug could have prevented them, and penicillamine should not be blamed for them. There are overwhelming arguments that this is not the case. First, the penicillamine-induced deteriorations are sudden, explosive worsenings within a few weeks, and do not follow the more gradual time course of each patient's history prior to diagnosis and treatment. An example is a 22-year-old man who presented elsewhere with tremor, drooling, and mild dysarthria. He was placed on penicillamine and over a 6-week period showed significant deterioration. His dysarthria became worse, his facial dystonia and drooling were worse, and he had developed dystonia of all limbs. He could barely be understood and was bedridden. At this 6 -week point in his penicillamine therapy, we saw him for the first time. Magnetic resonance imaging (MRI) of the brain at this time showed typical basal ganglia lesions but no white matter lesions. Because we thought the patient had already gone through penicillamine-induced worsening, we elected to leave him on penicillamine. Within another 4 weeks the patient had deteriorated further to the point of being anarthric and even less mobile in bed. He had also developed a seizure disorder. A repeat MRI showed new, severe white matter lesions. ${ }^{3}$ We have not seen such a rapid, explosive deterioration in brain MRI in numerous other neurologic patients we have followed who were not on penicillamine.

A second, overwhelming piece of evidence that the kind of deterioration induced by penicillamine is not part of the preordained natural history of the disease is that if the patients are treated with a different drug, this type of worsening does not occur. ${ }^{4-6}$ I will turn to preferred therapy for this type of patient subsequently in this article.

The reason penicillamine is so prone to induce neurologic worsening is not known with certainty. We have studied rats loaded with copper by high copper diets and found that during initial penicillamine treatment, there is a tendency for brain copper levels to increase further. Initial treatment with penicillamine causes a huge cupruresis, and the only way the copper can get to the kidneys is through the bloodstream. Therefore, one can speculate that during the movement of large amounts of copper, much of it from the liver, through the bloodstream, brain copper increases further, causing further toxicity. However, it can be hypothesized that penicillamine causes shifts in copper from various intraneuronal locales to others, causing toxicity in that manner, or there may be other mechanisms. Regardless of mechanism, it is clear that penicillamine therapy is a terrible risk in these patients.

What are the alternatives to penicillamine for the neurologically presenting patient? We have developed zinc acetate (Galzin ${ }$, Gate Pharmaceutical Co, Montgomeryville, PA, U.S.A.) for the maintenance therapy of Wilson's disease, and it was approved in January 1997 by the FDA for this purpose. ${ }^{7-23}$ Zinc has been extensively used by the Hoogenraad group in the Netherlands, ${ }^{24-26}$ and they use it for initial therapy in all patients with Wilson's disease. They have not reported any problems with this approach, but they also have not reported any detailed neurologic functional studies so that one can determine whether there has been some deterioration and if so, how much. I have personally started three neurologically presenting patients on zinc, and while two did well, one did have significant progression of tremor during the first few months of therapy. Zinc does not have the risk of causing explosive worsening by moving large amounts of copper around the body, but it is a slowacting decoppering agent, and the patient's disease can show some progression during the 4-6 months it takes zinc to reduce copper below the toxic threshold. For this reason, I am not enthusiastic about the use of zinc for initial therapy, but it is a better and safer choice than penicillamine.

Trientine is officially approved in the United States to use as an alternative to penicillamine when the patient is intolerant of penicillamine. Because of this restriction, it has not seen extensive use and there is little data on its risks of causing neurologic deterioration. We are aware of one unpublished patient who deteriorated on trientine, but because studies have not been published, there is no way of knowing the actual risk of deterioration on trien- 
tine. Because it is a chelator, it probably shares with penicillamine some risk, but because it is not a reductive chelator like penicillamine, its in vivo properties may be different. A study is needed to define the risk of initial treatment with trientine.

Over recent years we have developed tetrathiomolybdate (TM) for initial treatment of the neurologic patient. $^{4-6}$ TM is extremely fast acting yet safe, and because it acts through an entirely different mechanism, it does not share the risk with penicillamine of making patients deteriorate neurologically.

To study TM, we set up a detailed protocol that involved weekly quantitative evaluation of speech function and neurologic function other than speech. TM was given for an 8-week period. In all, 55 patients have been studied $^{4-6}$ (subsequent unpublished studies). Only two patients in this group showed any deterioration. One of these was a patient who deteriorated in the month or two after discharge and who we only treated for 6 weeks, a change we made with a few patients to reduce their time away from home. Since that result, we have gone back to an exclusive 8-week protocol. We do not know why the other patient deteriorated. In both cases, while the quantitative neurologic score exceeded our limits and was therefore deemed significant, the actual clinical deterioration was rather mild.

Most important, 53 of 55 patients (96\%) of carefully evaluated patients showed no evidence of deterioration. These patients were then placed on zinc maintenance therapy, and over the next 2 years, brain damage was slowly repaired. Within 1-2 years of initiating therapy, the recovery of these patients was generally remarkable. ${ }^{4-6}$ Mild patients generally showed complete recovery, moderately affected patients generally only had slight residual, and even some of the severely affected patients recovered enough to live a fairly normal life.

TM has shown no toxicity per se, but because it is so potent, with overtreatment it can render the bone marrow temporarily copper-deficient, leading to easily reversible bone marrow suppression. Stopping the drug temporarily quickly reverses this problem. We have seen this effect primarily with higher doses that we have used in the past. With our current dose (120 mg daily), such a response is rare.

The main disadvantage of TM is its availability. The drug needs to be especially prepared for human use, and it must be carefully stored, with air excluded, to maintain stability. Thus, TM is generally not accessible for occasional use. Our use is experimental and is an investigational new drug study filed with the FDA. Until now, we have not sought permission to share the drug with physicians elsewhere because the protocol was fairly com- plicated. With increasing experience and simplification of the protocol, we may be able to share the drug. For those patients who are capable of coming to our Medical Center, the clinical trial provides free care and hospitalization. We also have a recently funded grant, along with Dr. Michael Schilsky of Albert Einstein College of Medicine, to compare trientine and TM in the initial treatment of neurologically presenting Wilson's disease.

The initial treatment of patients presenting with liver disease requires a triage to determine whether the patient is unlikely to survive without liver transplantation. We use the prognostic index of Nazer and colleagues ${ }^{27}$ as one part of that determination. If medical therapy is elected, we use a combination of trientine and zinc. Trientine is used to get a relatively quick and sizable negative copper balance, and zinc is used, at least initially, for its induction of hepatic metallothionein, which will bind intrahepatic copper and theoretically reduce further copper damage. However, the best therapy or combination of therapies has not been established rigorously for this type of patient. For example, TM, which is life-saving in copper-poisoned sheep who otherwise die of hepatic failure, should be examined.

For maintenance therapy, which includes presymptomatic patients from the beginning and pediatric patients, the clear treatment of choice is zinc. Penicillamine has such a long list of acute, subchronic, and chronic toxicities that it should never be used in the treatment of Wilson's disease. ${ }^{23}$ Beyond the known toxicities are theoretical side effects not yet reported but which could be disastrous. For example, studies in rats and chickens have shown that penicillamine causes damage to collagen and elastin, including that of blood vessels. ${ }^{28,29}$ One worries about strokes, aneurysms, and the like in patients treated for a lifetime with penicillamine. Trientine appears to be less toxic than penicillamine for maintenance therapy but still has a list of significant toxicities.

In contrast, zinc used in maintenance therapy is free of side effects except for an occasional patient who has mild gastric intolerance of zinc, and this can usually be mitigated or eliminated by some simple maneuvers. ${ }^{22}$ We are aware that Dr. Walshe has treated patients who had problems with gastric intolerance, but he has used zinc sulfate rather than the zinc acetate we use, and the sulfate salt is more irritating to the stomach.

There have also been occasional reports of zinc failures, including one from Dr. Walshe. ${ }^{30,31}$ However, review of these cases always reveals that there are no data to indicate that the patient was actually taking their zinc prior to becoming ill. Because periodic noncompliance reaches as high as $30 \%$, even in our carefully followed and monitored patients, ${ }^{22}$ each of these cases is probably 
the result of noncompliance. Any Wilson's disease patient not taking anti-copper medication will deteriorate. Compliance with zinc therapy is easily checked by following 24-hour urine zinc and copper.

Finally, zinc is the treatment of choice during pregnancy in women with Wilson's disease. Zinc is not teratogenic, and we have followed 18 pregnancies on zinc therapy with generally good results. ${ }^{22}$ Penicillamine is teratogenic in animals and people,$^{32-34}$ and trientine is known to be teratogenic in animals. ${ }^{34}$

Returning to the main topic of this controversy, whether penicillamine should be used for initial therapy of the neurologically presenting patient with Wilson's disease, it turns out, unfortunately for Dr. Walshe's side of the argument, that there is no controversy at all. Penicillamine should not be used, pure and simple. It is far too dangerous, far too likely to produce a severely and permanently disabled patient. In contrast, TM looks good at preserving neurologic function and allowing most patients the opportunity to recover and return to a productive life. If TM is unavailable to a given patient, zinc or trientine are far safer and better choices for treating this type of patient.

\section{REFERENCES}

1. Walshe JM. Penicillamine. A new oral therapy for Wilson's disease. Am J Med 1956;21:487-495.

2. Walshe JM. Treatment of Wilson's disease with trientine (triethylene tetramine) dihydrochloride. Lancet 1982;1:643-647.

3. Brewer G, Terry CA, Aisen AM, Hill GM. Worsening of neurologic syndrome in patients with Wilson's disease with initial penicillamine therapy. Arch Neurol 1987;44:490-493.

4. Brewer GJ, Dick RD, Yuzbasiyan-Gurkan V, Tankanow R, Young $\mathrm{AB}$, Kluin KJ. Initial therapy of Wilson's disease patients with tetrathiomolybdate. Arch Neurol 1991;48:42-47.

5. Brewer GJ, Dick RD, Johnson V, et al. Treatment of Wilson's disease with tetrathiomolybdate. I: initial therapy in 17 neurologically affected patients. Arch Neurol 1994;51:545-554.

6. Brewer GJ, Johnson V, Dick RD, Kluin KJ, Fink JK, Brunberg JA. Treatment of Wilson's disease with ammonium tetrathiomolybdate. II: initial therapy in 33 neurologically affected patients and follow-up on zinc therapy. Arch Neurol 1996;53:1017-1025.

7. Brewer G, Hill GM, Prasad AS, Cossack ZT, Rabbani P. Oral zinc therapy for Wilson's disease. Ann Intern Med 1983;99:314-320.

8. Hill GM, Brewer GJ, Prasad AS, Hydrick CR, Hartmann DE. Treatment of Wilson's disease with zinc. I: oral zinc therapy regimens. Hepatology 1987;7:522-528.

9. Hill GM, Brewer GJ, Juni JE, Prasad AS, Dick RD. Treatment of Wilson's disease with zinc. II: validation of oral 64-copper uptake with copper balance. Am J Med Sci 1986;12:344-349.

10. Brewer GJ, Hill GM, Dick RD, et al. Treatment of Wilson's disease with zinc. III: prevention of reaccumulation of hepatic copper. J Lab Clin Med 1987;109:526-531.

11. Brewer GJ, Hill GM, Prasad AS, Dick RD. Treatment of Wilson's disease with zinc. IV: efficacy monitoring using urine and plasma copper. Proc Soc Exp Biol Med 1987;7:446-455.

12. YuzbasiyanGurkan V, Brewer GJ, Abrams GD, Main B, Giacherio D. Treatment of Wilson's disease with zinc. V: changes in serum levels of lipase, amylase and alkaline phosphatase in Wilson's disease patients. J Lab Clin Med 1989;114:520-526.
13. Brewer GJ, YuzbasiyanGurkan V, Lee DY, Appelman H. The treatment of Wilson's disease with zinc. VI: initial treatment studies. J Lab Clin Med 1989;114:633-638.

14. Lee DY, Brewer GJ, Wang Y. Treatment of Wilson's disease with zinc. VII: protection of the liver from copper toxicity by zincinduced metallothionein in a rat model. $J$ Lab Clin Med 1989;114: 639-645.

15. Brewer GJ, Yuzbasiyan-Gurkan V, Dick R. Zinc therapy of Wilson's disease. VIII: dose response studies. Journal of Trace Elements in Experimental Medicine 1990;3:227-234.

16. Brewer GJ, YuzbasiyanGurkan V, Johnson V. The treatment of Wilson's disease with zinc. IX: response of serum lipids. $J$ Lab Clin Med 1991;118:466-470.

17. YuzbasiyanGurkan V, Grider A, Nostrant T, Cousins RJ, Brewer GJ. The treatment of Wilson's disease with zinc. X: intestinal metallothionein induction. J Lab Clin Med 1992;120:380-386.

18. Brewer GJ, YuzbasiyanGurkan V, Johnson V, Dick RD, Wang Y. Treatment of Wilson's disease with zinc. XI: interaction with other anticopper agents. J Am Coll Nutr 1993;12:26-30.

19. Brewer GJ, YuzbasiyanGurkan V, Johnson V, Dick RD, Wang Y. Treatment of Wilson's disease with zinc. XII: dose regimen requirements. Am J Med Sci 1993;305:199-202.

20. Brewer GJ, Dick RD, Yuzbasiyan-Gurkan V, Johnson V, Wang Y. Treatment of Wilson's disease with zinc. XIII: therapy with zinc in presymptomatic patients from the time of diagnosis. J Lab Clin Med 1993;123:849-858.

21. Brewer GJ, Johnson V, Kaplan J. The treatment of Wilson's disease with zinc. XIV: studies of the effect of zinc on lymphocyte function. J Lab Clin Med 1997;129:649-652.

22. Brewer GJ, Dick RD, Johnson VD, Brunberg JA, Kluin KJ, Fink JK. The treatment of Wilson's disease with zinc. XV: long-term follow-up studies. J Lab Clin Med. 1998;132:264-278.

23. Brewer GJ, YuzbasiyanGurkan V. Wilson's disease. Medicine 1992;71:139-164.

24. Hoogenraad TU, Koevoet R, De Ruyter Korver EGWM. Oral zinc sulfate as long-term treatment in Wilson's disease (hepatolenticular degeneration). Eur Neurol 1979;18:205-211.

25. Hoogenraad TU, Van den Hamer CJA, Koevoet R, De Ruyter Korver EGWM. Oral zinc in Wilson's disease. Lancet 1978;ii: $1262-1263$.

26. Hoogenraad TU, Van Hatum J, Van den Hamer CJA. Management of Wilson's disease with zinc sulfate. Experience in a series of 27 patients. J Neurol Sci 1987;77:137-146.

27. Nazer H, Ede RJ, Mowat AP, Williams R. Wilson's disease: clinical presentation and use of prognostic index. Gut 1986;27:13771381.

28. Nimni ME, Bavetta LA. Collagen defect induced by penicillamine. Science 1965;150:905-907.

29. Roncheti IP, Fornieri C, Contri MB, Quaglino D Jr, Caselgrandi E. Effect of DL-penicillamine on the aorta of growing chickens. $\mathrm{Am}$ J Clin Pathol 1986;124:436-447.

30. Shimon I, Sela BA, Moses B, Doley E. Hemolytic episode in a patient with Wilson's disease treated with zinc. Isr J Med Sci 1993;29:646-647.

31. Walshe JM, Munro NAR. Zinc-induced deterioration in Wilson's disease aborted by treatment with penicillamine, dimercaprol, and a novel zero copper diet. Arch Neurol 1995;52:10-11.

32. Mjolnerod OK, Dommerud SA, Rasmussen K, Gjeruldsen ST. Congenital connective tissue defect probably due to Dpenicillamine treatment in pregnancy. Lancet 1971;i:673-675.

33. Solomon L, Abrams G, Dinner M, Berman L. Neonatal abnormalities associated with D-penicillamine treatment during pregnancy. N Engl J Med 1977;296:54-55.

34. Keen CL, Lonnerdal B, Hurley LS. Teratogenic effect of copper deficiency and excess. In: Sorenson JRJ, ed. Inflammatory Disease and Copper. Clifton, NJ: Humana Press, 1982:109-121. 